\title{
Impact of COVID-19 Pandemic on Drug Overdoses in Indianapolis
}

\author{
Nancy Glober $(\mathbb{D} \cdot$ George Mohler • Philip Huynh • \\ Tom Arkins • Dan O'Donnell • Jeremy Carter • Brad \\ Ray
}

Accepted: 4 September 2020/Published online: 1 October 2020

(C) The New York Academy of Medicine 2020
Keywords Overdose - Opioid · COVID-19 · Pandemic . EMS

\section{Introduction}

The overdose epidemic persists as one of the most pressing public health issues in the United States (U.S.). The majority of deaths from overdoses are opioid-related, though the specific type of opioid varies [1-3]. Over time, increases in overdoses were initially linked to opioid pain analgesics but shifted to illicit opioids, such as heroin and fentanyl [2, 4-7]. More recently, public health officials and healthcare providers have noted a sharp increase in overdose deaths associated with illicit synthetic substances, specifically cocaine and methamphetamines, in urban areas [6-8].

Some evidence suggests that spikes have occurred in opioid overdoses in several jurisdictions across the U.S. during the COVID-19 pandemic [9-18]. To date, no national data exist to assess the impact to overdose events of the COVID-19 pandemic. Different theories exist to explain the observed increase in overdose deaths during the COVID-19 pandemic. Drug trafficking patterns likely changed secondary to disrupted travel and international social distancing guidelines. With the changes in drug trafficking, it is possible that illicit drugs differing in purity and potency are being manufactured locally and by individuals. In light of widespread social distancing, individual patterns of drug use may have changed. Drug users may be using drugs alone more often and using 
drugs more frequently. Rising unemployment rates may also drive up the frequency of drug use.

Using emergency medicine calls for service (CFS) and suspected accidental drug overdose deaths from the same jurisdiction (Marion County, Indiana), we comprehensively examine changes in overdose deaths after COVID-19 stay-at-home measures were implemented. Specifically, we analyze differences in the daily rate of CFS, including overdose CFS and CFS in which naloxone was administered, and the weekly rate of overdose deaths before and after the stay-at-home order in Indianapolis.

\section{Data and Analytic Method}

The data used in this study comes from Marion County, Indiana, home to Indianapolis, the state capital, and the 17 th most populous city in the U.S. We analyze four types of events: (1) all Indianapolis Emergency Medical Services (IEMS) calls for service (CFS), (2) the subset of calls for service where with primary or secondary provider impression was overdose, (3) the subset of IEMS calls for service where naloxone was administered, and (4) fatal overdose events (data provided by the Marion County Coroner's Office, which contain all suspected accidental overdose deaths). For those events pending toxicology testing, circumstantial information such as scene investigation, medical history, and social history from the coroner's reports was used to determine deaths likely secondary to drug overdose. Data on CFS including total number of CFS, provider impression, and naloxone administration was abstracted from the IEMS medical record system, ESO. Most EMS CFS in which naloxone was administered had a provider impression of overdose. However, in some overdose CFS, naloxone was not administered as it was not indicated by the IEMS protocol, which only directs naloxone administration for respiratory depression [19].

For this study, we examined data from January 1, 2019 through July 24, 2020. The stay-at-home order for Indiana started on 11:59 p.m. March 24, 2020. We perform 2 sample $t$ tests to detect a difference in means of the daily rate of each event type before and after the initial stay-at-home order date (for overdose deaths, we analyze weekly rate). We separately consider two different pre-pandemic periods: (1) the 122-day period directly preceding the stay-at-home order (matching the 122-day period after the order) and (2) the period from March 25, 2019 to July 24, 2019 (to assess the robustness of the $t$ test against seasonal effects).

The rationale behind using the $t$ test is that, under the null hypothesis, the daily rate of events has the same mean before and after the stay-at-home order went into effect. However, the $t$ test additionally assumes that daily event counts are independent. Urban event data can exhibit autocorrelation and seasonal effects that can also produce a temporary change in the rate of events. To assess whether this is the case, we additionally fit autoregressive integrated moving average (ARIMA) forecasting models [20] to the time series of daily event counts leading up to 11:59 p.m. March 24, 2020. We then inspected the accuracy of the forecast over the period March 25, 2020 to July 24, 2020. Death data was only available through July 7, 2020. ARIMA model parameters were selected using the Akaike Information Criterion [21]. Statistical analyses were performed in R.

\section{Results}

In Table 1, we display the results for the $t$ test for a difference in the daily rate of events before and after the stay-at-home order (pre-period defined as period directly preceding March 25, 2020). We observe rate increases of $61.0 \%$ (naloxone administration) and $43.0 \%$ (primary or secondary EMS provider impression of overdose) that are both significant. Drug overdose deaths are also higher by $47.0 \%$. Overall calls for service were up by $4.0 \%$.

In Table 2, we display results for the $t$ test for a difference in the daily rate of events before and after the stay-at-home order, where the pre-period is instead defined as March 25, 2019 to July 24, 2019 (to assess for seasonal variation). In this case, we observe rate increases of $90.0 \%$ (naloxone administration), $30.0 \%$ (primary or secondary EMS provider impression of overdose), and 104.0\% (overdose deaths). In the second $t$ test, the difference in means of total daily CFS is not statistically significant.

To assess the robustness of our results against the independence assumptions of the $t$ test, we fit autoregressive integrated moving average (ARIMA) forecasting models to the time series of daily event counts leading up to the shut-down. We then inspect the accuracy of the forecast over the period March 25, 2020 to July 24, 2020. In Fig. 1, we observe that the time series of overdoses in which naloxone was administered and all overdose CFS are well above the $99 \%$ confidence interval of the forecasted daily rate of events from 
Table 1 Two sample $t$ tests for a difference in means in the daily rate of events pre- and post-stay-at-home order. The pre-period is defined as the 122-day period directly preceding the stay-at-home order on March 25, 2020.

\begin{tabular}{|c|c|c|c|c|c|}
\hline & Mean (pre) & Mean (post) & Std. err & $t$ stat. & $p$ value \\
\hline Overdose (naloxone administered) & 6.4 & 10.3 & 0.434 & 8.989 & $<0.001$ \\
\hline Overdose (all EMS provider impression of overdose) & 10.6 & 15.2 & 0.552 & 8.286 & $<0.001$ \\
\hline All EMS & 321.0 & 334.5 & 3.384 & 3.979 & $<0.001$ \\
\hline Overdose deaths* & 8.4 & 12.4 & 1.326 & 2.971 & 0.006 \\
\hline
\end{tabular}

*Weekly instead of daily rate

mid-April through mid-July. We note that the rate of overdose CFS increases between implementation of the stay-at-home order and staged reopening in Indiana. Even after the staged reopening, CFS for overdose and CFS in which naloxone was administered persisted above last year's levels. In the case of deaths from overdoses, there is a several-week period in between the stay-at-home order and full reopening when the weekly event rate is above the $99 \%$ confidence bounds of the ARIMA forecast. The observed time series of all EMS CFS, on the other hand, is well within the $99 \%$ confidence bounds of the ARIMA forecast.

In Table 3, we display counts of EMS overdose CFS, disaggregated by age, race/ethnicity, and zip code, before and after the stay-at-home order. We observe that the percentage of overdose CFS of each particular age group does not significantly change before or after the stay-at-home order. For example, overdose calls where the individual is below 30 years of age comprise $33.9 \%$ of overdose calls before the stay-at-home order and $30.3 \%$ of overdose calls after. We also do not observe significant effects due to race/ethnicity or zip code.

\section{Discussion}

We used data from the EMS electronic medical records and the coroner's office to measure changes in the rates of overdose CFS and deaths after implementation of the
COVID-19 stay-at-home order. The increases we observed-CFS in which naloxone was administered and overdose deaths - are consistent with recent studies $[16,22]$. Looking at several micro datasets in the same jurisdiction, our study additionally revealed that increases in overdoses were consistent across age and racial/ethnic groups, as well as spatially in high-volume zip codes.

The COVID-19 pandemic is rapidly changing, as is the social response. The data reported in this analysis come from a major U.S. city 122 days after the stay-athome order. These results might look different as time elapses, in different jurisdictions, or with different overdose measures; to that end, we have provided the necessary steps for replication or model specification.

Interestingly, while rates of overdose-related CFS and deaths increased after the stay-at-home order was implemented, they continued to increase after the staged reopening began. The trend in the data suggests possible early dissipation of the increase in opioid-related calls and deaths as statewide reopening continues. This suggests that the increases we observe are secondary to social distancing. Furthermore, the spike appears consistent across rage, age, and space, suggesting similar patterns of abuse to pre-COVID-19 isolation, but with increasing intensity. Law enforcement data may be able to offer further insight into changes in illicitly trafficked substances and whether it mirrored the overdose rates we appreciated.

Table 2 Two sample $t$ tests for a difference in means in the daily rate of events before and after the stay-at-home order. The pre-period is defined as March 25, 2019 to July 24, 2019

\begin{tabular}{|c|c|c|c|c|c|}
\hline & Mean (pre) & Mean (post) & Std. err & $t$ stat. & $p$ value \\
\hline Overdose (naloxone) & 5.4 & 10.3 & 0.440 & 11.089 & $<0.001$ \\
\hline Overdose (all) & 11.7 & 15.2 & 0.595 & 5.839 & $<0.001$ \\
\hline All EMS & 333.3 & 334.5 & 3.563 & 0.338 & 0.736 \\
\hline Overdose deaths* & 6.1 & 12.4 & 1.179 & 5.359 & $<0.001$ \\
\hline
\end{tabular}

*Weekly instead of daily rate 

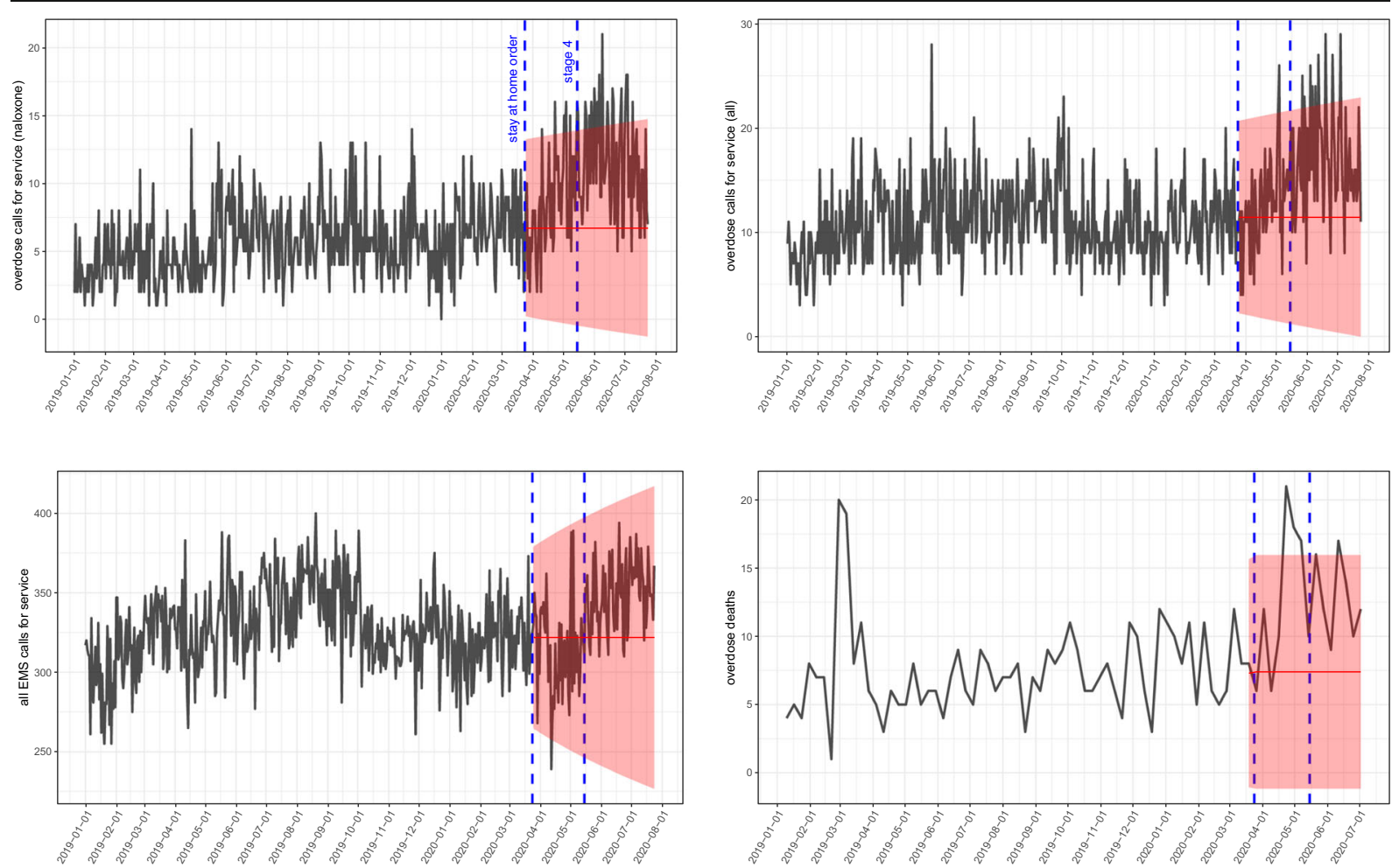

Fig. 1 Daily observed EMS CFS (black curve) in which naloxone was administered (top left), daily rate of all overdose CFS (top right), daily rate of all EMS CFS (lower left), and drug overdose deaths per week (lower right). Blue vertical lines indicate the stayat-home order date (March 25, 2020) and the beginning of

reopening in Indianapolis (May 16, 2020). Red shaded region indicates $99 \%$ confidence interval for ARIMA forecast over the period after the stay-at-home order (estimated using data up through March 24, 2020)

Table 3 Counts of EMS overdose calls for service, disaggregated by age, race/ethnicity and zip code (top 5 by volume), before and after the stay-at-home order on March 25, 2020

\begin{tabular}{|c|c|c|c|c|c|}
\hline & & Count (pre) & $\%$ (pre) & Count (post) & $\%$ (post) \\
\hline \multirow[t]{5}{*}{ Age } & $<30$ & 1654 & 33.9 & 556 & 30.3 \\
\hline & $30-40$ & 1481 & 30.4 & 624 & 34.0 \\
\hline & $40-50$ & 843 & 17.3 & 313 & 17.0 \\
\hline & $50-60$ & 585 & 12.0 & 235 & 12.8 \\
\hline & $60+$ & 313 & 6.4 & 108 & 5.9 \\
\hline \multirow[t]{3}{*}{ Race/Ethn. } & Black & 1479 & 30.2 & 494 & 26.6 \\
\hline & Other & 141 & 2.9 & 51 & 2.7 \\
\hline & White & 3274 & 66.9 & 1314 & 70.7 \\
\hline \multirow[t]{6}{*}{ Zip code } & 46201 & 568 & 11.6 & 221 & 11.9 \\
\hline & 46203 & 400 & 8.2 & 153 & 8.2 \\
\hline & 46204 & 226 & 4.6 & 61 & 3.3 \\
\hline & 46218 & 262 & 5.4 & 128 & 6.9 \\
\hline & 46219 & 311 & 6.4 & 108 & 5.8 \\
\hline & Other & 3127 & 63.9 & 1188 & 63.9 \\
\hline
\end{tabular}


It is worth noting that although we focused on patient-centered factors, response factors may have played a role in some of the increases we saw. Multiple reports have noted that law enforcement was reluctant to administer naloxone during the COVID-19 pandemic $[23,24]$. As law enforcement is often the first to arrive on the scene, fewer administrations of naloxone by law enforcement may have led to some inflation in the rates of naloxone administration by EMS [25].

Our study is not without limitations. We are using routinely collected EMS electronic medical record data as surrogate measures of overdose and opioidrelated overdose. Moreover, our fatal overdose data are suspected overdose events but lack confirmation with toxicological testing. Although we do not have the toxicology results on the overdose deaths, recent published rates suggest more than $80 \%$ of accidental overdose deaths are opioid-related [26]. Furthermore, while this study highlights changes in overdoses and naloxone administration by EMS, it does not attempt to describe changes in volumes of patients who overdose but do not interact with the EMS system. Naloxone is often prescribed upon discharge from the hospital and can be readily purchased at pharmacies. This study does not capture home naloxone use without a subsequent EMS CFS. Despite these limitations, our study presents data, methods, and analysis that can be replicated with similar secondary data in other EMS jurisdictions.

\section{Conclusion}

Experts suggest an imminent second surge of COVID19 in the U.S. Frontline treatment providers should be engaged in mitigation of overdose events that increase during the stay-at-home order. Our data supports implementation of critical public health and harm reduction strategies for substance use, particularly during times of social isolation and subsequent reopening. Those should include widely available naloxone and buprenorphine with telehealth follow-up.

Acknowledgements This work was supported in part by NSF grants ATD-1737996 and SCC-1737585 and, in part, by the Centers for Disease Control and Prevention Overdose Data to Action (CDC; Grant\#NU17CE924990).

\section{References}

1. Gladden RM. Changes in opioid-involved overdose deaths by opioid type and presence of benzodiazepines, cocaine, and methamphetamine-25 states, July-December 2017 to January-June 2018. MMWR Morb Mortal Wkly Rep. 2019;68 https://doi.org/10.15585/mmwr.mm6834a2.

2. Hedegaard H. Drug overdose deaths in the United States, 1999-2018. MD: National Center for Health Statistics, Hyattsville. 2020;356:8.

3. Wilson CG, Ramage M, Fagan EB. A primary care response to COVID-19 for patients with an opioid use disorder. $J$ Rural Health. 2020; https://doi.org/10.1111/jrh.12438.

4. Centers for Disease Control \& Prevention. Opioid overdose: understanding the epidemic. 2020. https://www.cdc. gov/drugoverdose/epidemic/index.html. Accessed 1 June 2020.

5. Ciccarone D. The triple wave epidemic: supply and demand drivers of the US opioid overdose crisis. Int $J$ Drug Policy. 2019;71:183-8. https://doi.org/10.1016/j. drugpo.2019.01.010.

6. Hainer R. Polysubstance use: a dangerous fourth wave in the opioid crisis: Boston Medical Center; 2019. https://www.bmc.org/healthcity/populationhealth/polysubstance-use-dangerous-fourth-waveopioid-crisis. Accessed 1 June 2020.

7. Wilson N. Drug and opioid-involved overdose deathsUnited States, 2017-2018. MMWR Morb Mortal Wkly Rep. 2020;69 https://doi.org/10.15585/mmwr.mm6911a4.

8. Hoots B, Vivolo-Kantor A, Seth P. The rise in non-fatal and fatal overdoses involving stimulants with and without opioids in the United States. Addiction. 2020;115(5):946-58. https://doi.org/10.1111/add.14878.

9. Alter A, Yeager C. The consequences of COVID-19 on the overdose epidemic: overdoses are Increasing (p. 5). Washington/Baltimore High Intensity Drug Trafficking Area. 2020 http://www.odmap. org/Content/docs/news/2020/ODMAP-Report-May2020.pdf. Accessed 1 June 2020.

10. Bellrichard C. B.C. moves to "safe supply" as overdose deaths spike during COVID-19 pandemic | CBC News. CBC. 2020 https://www.cbc.ca/news/indigenous/bc-safesupply-opioid-pandemic-1.5565081. Accessed 1 June 2020.

11. Huechtker S. As COVID-19 pandemic grows, so does South Carolina's opioid problem [news]. WRDW. 2020 https://www.wrdw.com/content/news/As-COVID-19pandemic-grows-so-does-South-Carolinas-opioid-problem570430711.html. Accessed 1 June 2020.

12. Kirkland T. Overdose deaths skyrocket in Pennsylvania during COVID-19 pandemic. WHP. $2020 \mathrm{https} / / /$ local21 news.com/news/local/overdose-deaths-skyrocket-inpennsylvania-during-covid-19-pandemic. Accessed 1 June 2020.

13. Local 24. Spike in drug overdoses in Shelby County over past month. Localmemphis.Com. 2020 https://www. localmemphis.com/article/news/health/spike-drugoverdoses-shelby-county-over-past-month/522-347077cd2933-4396-9c66-f1d1ad02894a. Accessed 1 June 2020.

14. mycitizensnews. Number of fatal overdoses spike in state. Citizen's News. 2020 https://www.mycitizensnews. 
net/news/number-of-fatal-overdoses-spike-in-state/. Accessed 1 June 2020.

15. Roth AN. An upstate New York county sees a spike in drug overdoses. Is there a COVID-19 connection? Rochester Democrat and Chronicle. $2020 \mathrm{https}$ ://www. democratandchronicle.com/story/news/2020/04/14/covid19-pandemic-exacerbating-heroin-epidemic-advocatessay/2988453001/. Accessed 1 June 2020.

16. Slavova S, Rock P, Bush HM, Quesinberry D, Walsh SL. Signal of increased opioid overdose during COVID-19 from emergency medical services data. Drug Alcohol Depend. 2020;214:108176. https://doi.org/10.1016/j. drugalcdep.2020.108176.

17. Struett D. DuPage sees 'unusual spike' in overdose deaths during coronavirus pandemic: coroner. Chicago Sun-Times. $2020 \mathrm{https}$ //chicago.suntimes.com/2020/5/13/21257820 /dupage-opioid-overdose-death-coronavirus-coronerrichard-jorgensen-covid-19. Accessed 1 June 2020.

18. Volkow ND. Collision of the COVID-19 and addiction epidemics. Ann Intern Med. 2020;173:61-2. https://doi. org/10.7326/M20-1212.

19. Indianapolis Emergency Medical Services. Indianapolis metropolitan area EMS protocols: 2014 version. Indianapolis Emergency Medical Services. Indianapolis: Indiana; 2014.

20. Hyndman RJ, Khandakar Y. Automatic time series for forecasting: the forecast package for $R$ (issue 6/07). Monash University, Department of Econometrics and Business Statistics. Innsbruck: Austria; 2007
21. Akaike H. A new look at the statistical model identification. IEEE Trans Autom Control. 1974;19(6):716-23. https://doi. org/10.1109/TAC.1974.1100705.

22. American Medical Association. Issue brief: reports of increases in opioid-related overdose during COVID pandemic. Chicago: Illinois; 2020.

23. Feder S. Overdose calls are rising, but some police in Indiana and Texas have been banned from giving a "life-saving" drug. Business Insider. 2020 https://www.businessinsider. com/indiana-and-texas-police-officers-no-longer-equippedwith-naloxone-2020-4. Accessed 1 June 2020.

24. Schladebeck J. Indiana police department suspends Narcan use as officials raise alarm about overdose deaths during coronavirus pandemic. Daily News. 2020 https://www. nydailynews.com/coronavirus/ny-coronavirus-indianapolice-department-suspension-narcan-backlash-20200416v2qddgofrvbltfzumjat7ij6rm-story.html. Accessed 1 June 2020.

25. Fisher R, O’Donnell D, Ray B, Rusyniak D. Police officers can safely and effectively administer intranasal naloxone. Prehosp Emerg Care. 2016;20(6):675-80.

26. Ray B, Lowder E, Bailey K, Huynh P, Benton R, Watson D. Racial differences in overdose events and polydrug detection in Indianapolis, Indiana. Drug Alcohol Depend. 2020;206: 107658. https://doi.org/10.1016/j.drugalcdep.2019.107658.

Publisher's Note Springer Nature remains neutral with regard to jurisdictional claims in published maps and institutional affiliations. 